\title{
Características dos Programas escolares de Prevenção ao Uso de Drogas no Brasil
}

\author{
Characteristics of school-based drug prevention programs in Brazil
}

Ana Paula Dias Pereira (https://orcid.org/0000-0001-9944-4004) ${ }^{1}$

Zila M Sanchez (https://orcid.org/0000-0002-7427-7956) ${ }^{1}$

${ }^{1}$ Departamento de Medicina Preventiva, Universidade Federal de São Paulo. R. Botucatu 740, Vila Clementino, Campus São Paulo. 04023-062 São Paulo SP Brasil. psiapdias@gmail.com

\begin{abstract}
The aim of this study was to identify the main characteristics of school-based drug prevention programs in Brazil and verify whether these interventions apply the prevention principles suggested by the National Institute on Drug Abuse (NIDA). A cross-sectional study was conducted using a random national sample of 1,151 public and private school managers. The data were collected using an online questionnaire. Poisson regression was used to identify factors associated with the application of a greater number of prevention principles in the programs. The findings showed that programs were generally sporadic, had an average duration of one semester, incorporated different program models, and primarily directed at students. The most active organization in the delivery of programs was the Military Police. Private schools were shown to be $14 \%$ more likely to apply more good practice principles than public schools. Furthermore, programs delivered by school staff, health institutions, or departments of education were more likely to apply more prevention principles. Efforts are needed to improve drug prevention practice in Brazilian schools. Our findings show that, overall, school-based drug prevention programs do not apply the NIDA prevention principles.
\end{abstract}

Key words Prevention, Drugs, Programs, School Health
Resumo $O$ objetivo deste artigo é identificar as características dos programas de prevenção ao uso de drogas desenvolvidos nas escolas brasileiras e verificar a existência de princípios de boas práticas de prevenção nestes programas. Estudo transversal com uma amostra probabilística de 1.151 dirigentes de escolas públicas e privadas. Os dados foram coletados por meio de um questionário online. Regressão de Poisson permitiu identificar os fatores associados à maior quantidade de princípios de boas práticas de prevenção nos programas. Os programas se operacionalizam sem regularidade e com duração média de um trimestre, dirigidos principalmente aos alunos, com integração de diferentes modelos teóricos e promovidos principalmente pela Polícia Militar. As escolas privadas possuem $14 \%$ mais chance de apresentarem mais princípios de boas práticas em seus programas, quando comparadas às escolas públicas. Programas ofertados por uma equipe da própria escola ou por uma instituição da área de saúde ou pela secretaria de educação possuem maiores chances de apresentarem mais princípios de boas práticas. As ações de prevenção ao uso drogas nas escolas brasileiras necessitam de aperfeiçoamento das medidas adotadas. Os programas não se baseiam em evidências científicas e boas práticas em prevenção. Palavras-chave Prevenção, Drogas, Programas, Saúde Escolar 


\section{Introdução}

Programas de prevenção são comumente considerados estratégias apropriadas e efetivas para prevenir o uso de drogas no ambiente escolar tendo como objetivos criar novos fatores de proteção e reduzir os fatores de risco dos alunos em idade escolar ${ }^{2,3}$. Embora muitos estudos apresentem resultados positivos na capacidade dos programas escolares em reduzir ou retardar o consumo de álcool e outras drogas ${ }^{4,5}$, outros estudos têm evidenciado potencial efeito iatrogênico de alguns programas escolares de prevenção do uso de drogas ${ }^{6}$.

Estudos do campo da ciência da prevenção sugerem que as ações de prevenção ao uso de drogas nas escolas devem ser baseadas em evidências científicas, ou seja, que tenham apresentado resultados positivos em estudos de avaliação de eficácia ou efetividade, economizando, assim, recursos financeiros e humanos investidos em ações de pouco ou nenhum efeito ${ }^{2,4,7}$. No entanto, a prática de oferecer programas baseados em evidência é um desafio em diversos países ${ }^{8}$.

Revisões sistemáticas sobre as características dos programas de prevenção ao uso de drogas que apresentaram resultados positivos identificaram ações como: o uso de métodos interativos, seções estruturadas, intervenções aplicadas por profissionais treinados, intervenções que proporcionam oportunidades para praticar e aprender habilidades pessoais e sociais e que contemplam múltiplas dimensões como comunidade e famí$\operatorname{lia}^{9,10}$.

O National Institute of Drug Abuse (NIDA) ${ }^{11}$ apresenta os princípios de boas práticas em prevenção identificados com bases nas principais evidências científicas sobre componentes de programas que, quando presentes, favorecem o aparecimento de resultados positivos, ou seja, redução no uso de drogas entre os participantes. Dessa forma, é sugerido que os profissionais que atuam no campo da prevenção se orientem pelas recomendações de boas práticas, a fim de que as ações desenvolvidas tenham maior chance de sucesso, por estarem alicerçadas em componentes comuns em programas efetivos ou eficazes em contexto internacional ${ }^{12}$.

No Brasil, pouco se sabe sobre as características dos programas de prevenção ao uso de drogas que estão sendo realizados nas escolas e se os componentes básicos de boas práticas de prevenção estão presentes nessas intervenções. Nesse sentido, é fundamental que esses progra- mas sejam identificados e compreendidos em sua composição através de um diagnóstico, a fim de favorecer o avanço da prevenção segura nas escolas brasileiras.

Assim, o presente estudo tem como objetivos identificar as características dos programas de prevenção ao uso de drogas desenvolvidos nas escolas brasileiras e verificar a existência de princípios de boas práticas de prevenção nesses programas.

\section{Método}

Foi realizado um estudo transversal com uma amostra probabilística de dirigentes das escolas públicas e privadas das cinco regiões brasileiras (Sul, Sudeste, Norte, Nordeste e Centro-Oeste).

\section{Amostra}

A população-alvo do estudo foi a de dirigentes de escolas públicas e privadas de ensino fundamental II e/ou ensino médio, localizadas nas regiões urbanas, registradas no Cadastro $\mathrm{Na}$ cional das Escolas de Educação Básica, Censo Escolar de 2012, fornecido pelo Instituto Nacional de Estudos e Pesquisas Educacionais (INEP). A amostra foi recrutada através de sorteio aleatório simples da população-alvo, via Excel (RAND) das escolas, mantendo a proporcionalidade do sorteio e das substituições de acordo com o universo amostral de cada região e cada rede de ensino, tornando, assim, a amostra autoponderada.

Para o cálculo do tamanho da amostra, considerou-se o universo finito de escolas ( $\mathrm{n}=$ 52.065), nível de confiança de 95\%, um erro absoluto de 3\% e uma distribuição de respostas de $50 \%$ (por não haver dados prévios sobre a prevalência de programas de prevenção nas escolas brasileiras), o que gerou uma amostra de 1.046 escolas. Considerando-se as potenciais perdas amostrais comuns em estudos com coleta de dados via internet ${ }^{13,14}$, optou-se por um sorteio de 2.090 escolas, para que fosse garantido, mesmo após as potenciais perdas e substituições, o tamanho amostral calculado.

Conforme apresentado na Figura 1, foram considerados para o presente estudo 1.151 questionários válidos, dos quais $580(51,1 \%)$ registraram que a escola possuía um programa de prevenção ao uso de drogas inserido no projeto político-pedagógico da escola. 


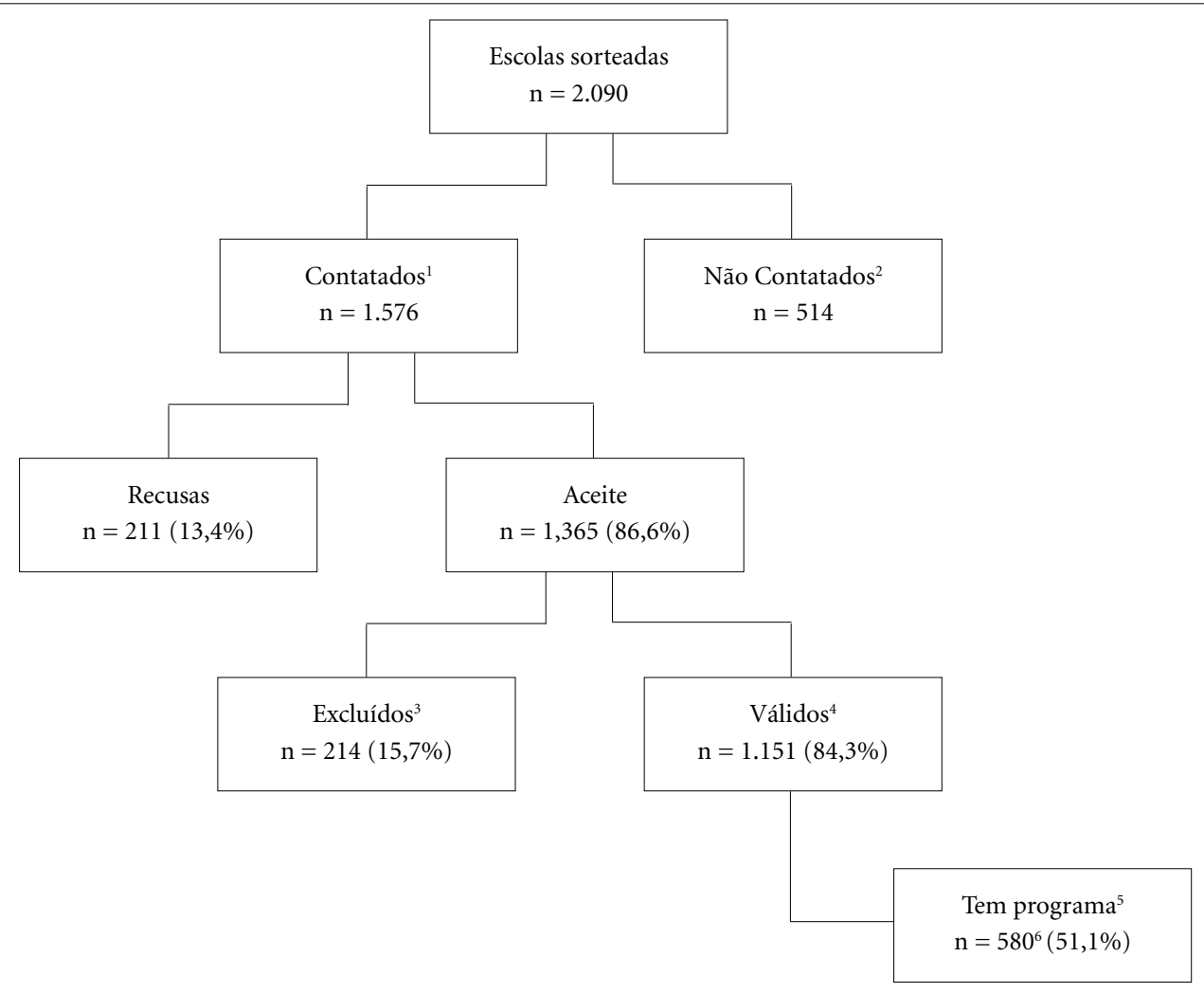

Figura 1. Fluxograma da amostra do estudo. Brasil, 2014.

${ }^{1}$ Contatos telefônicos e/ou por e-mail concluídos; ${ }^{2}$ Contatos telefônicos e/ou por e-mail não realizadas após diversas tentativas; ${ }^{3}$ Questionários com menos de $30 \%$ das questões preenchidas foram excluídos das análises; ${ }^{4}$ Questionários analisados; ${ }^{5} 15$ missing's - base de cálculo da porcentagem $n=1.136 ;{ }^{6}$ Questionários analisados que responderam possuir um programa de prevenção ao uso de drogas.

\section{Coleta de dados}

Os dirigentes inicialmente foram convidados a participar do estudo por mensagem enviada para os endereços eletrônicos das escolas. Utilizou-se para o envio dos e-mails o programa SurveyMonkey, que permitiu encaminhar mensagens a todos os endereços eletrônicos simultaneamente. Os entrevistados que não responderam à pesquisa, após 4 envios de e-mails, foram contatados por telefone. Os dados foram coletados no ano letivo de 2014.

\section{Instrumentos e variáveis}

Utilizou-se um questionário fechado, de autopreenchimento e anônimo, com 45 questões que avaliaram: características do entrevistado; característica da escola; educação em saúde na escola; capacitação dos respondentes na área de drogas e os princípios de boas práticas de prevenção ao uso de drogas do NIDA. Algumas das questões foram extraídas do questionário de Ringwalt et al. ${ }^{15} \mathrm{e}$ outras questões foram criadas para responder à necessidade de compreensão das características dos programas brasileiros e testadas em sua compreensão durante estudo prévio realizado na cidade de São Paulo ${ }^{16}$.

As variáveis referentes às características dos dirigentes participantes foram sexo; idade; escolaridade; cargo; participação em curso sobre drogas. As características demográficas da escola avaliadas foram: rede de ensino; região do Brasil; localização e porte da escola. Sobre os programas desenvolvidos na escola avaliou-se a frequência anual de aplicação; período de duração; público -alvo; nível de ensino ao qual o programa era oferecido; entidades promotoras; modelos teóricos; tipo de atividades desenvolvidas na escola sobre o tema drogas. 
Por fim, foi avaliada a presença de boas práticas de prevenção, através dos 15 princípios sugeridos pelo NIDA ${ }^{11}$ : focar na redução de fatores de risco e fortalecimento dos fatores de proteção ao uso de drogas; tratar de todas as drogas; lidar com o uso de drogas dos estudantes da escola; ajuste para atingir a idade e características pessoais do grupo de estudantes da escola; participação da família; participação da comunidade; atividades que são trabalhadas desde a pré-escola para lidar com agressividade, habilidades sociais e dificuldades escolares; para o ensino fundamental trabalhar treinamento de habilidades como: autocontrole, reconhecimento das emoções, comunicação, solução de problemas e competências acadêmicas; para o ensino médio envolve: suporte acadêmico, hábitos de estudo, comunicação, relacionamento com os pares, autoeficácia e assertividade, habilidades para a resistência à oferta de drogas, reforço de atitudes antidrogas; reforço do programa de prevenção em momentos de transição da vida, como por exemplo, no $9^{\circ}$ ano do ensino fundamental e no $3^{\circ}$ ano do ensino médio; combinação de mais de uma dimensão de prevenção, ou seja, envolver programa escolar, familiar e comunitário; programa é baseado em outro programa cuja eficácia já foi comprovada cientificamente; programa é contínuo e de longo prazo, atingindo diversas séries em diversos momentos do ano; inclui treinamento de professores para saber lidar com a turma em sala de aula; utiliza técnicas interativas de atividades: workshops, roda de discussões, teatralização e dinâmicas de grupo.

\section{Análise de dados}

$\mathrm{Na}$ análise descritiva, as variáveis qualitativas foram resumidas em frequências absolutas, prevalência e intervalos de confiança de $95 \%$. Para a variável numérica, número de princípios de boas práticas de prevenção, os dados foram apresentados em média, mínimo/máxima e desvio padrão. $\mathrm{Na}$ análise inferencial, foi utilizada a regressão de Poisson para avaliar os fatores associados à maior quantidade de princípios de boas práticas de prevenção nos programas, reportadas pelos dirigentes, considerando como variável dependente (desfecho) a soma das 15 variáveis de princípios de boas práticas de prevenção que podiam, assim, gerar uma pontuação total de boas práticas que potencialmente variaria entre 0 e 15.

As variáveis independentes (explicativas) analisadas foram as relacionadas às características das escolas (rede, região, porte e localização) e entidades promotoras dos programas de prevenção ao uso de drogas. A partir de um modelo inicial com as variáveis com $\mathrm{p}<0,20$, as variáveis sem significância estatística foram excluídas de maneira decrescente de acordo com o valor de $\mathrm{p}$, passo a passo, até se chegar a um modelo final, apenas com as variáveis significantes a $5 \%$. Os dados da regressão de Poisson são apresentados em IRR (incidence rate ratio) e intervalo de confiança de 95\% (IC95\%). Todas as análises foram realizadas com o programa Stata 13. Nas análises não foram utilizados pesos amostrais de correção, pois a amostra foi construída de maneira autoponderada, ou seja, mantendo a proporção de escolas sorteadas de acordo com o universo de cada região do Brasil, através de sorteio aleatório simples por região. As recusas foram proporcionais em todas as regiões.

\section{Aprovação Ética}

Todos os procedimentos éticos da pesquisa foram aprovados pelo Comitê de Ética em Pesquisa (CEP) da Universidade Federal de São Paulo. O Termo de Consentimento Livre e Esclarecido (TCLE) foi obtido de todos os participantes do estudo.

\section{Resultados}

A Tabela 1 mostra as características dos respondentes e das escolas do estudo. Observou-se predominância de escolas que pertenciam à rede pública de ensino, de porte pequeno, localizadas na região Sudeste e nos municípios que não são capitais de estados brasileiros. A maioria dos participantes era diretores, do sexo feminino, na faixa etária de 40 a 49 anos e com nível de ensino de pós-graduação.

Do total de participantes, $51,1 \%$ declararam que suas escolas possuíam programa de prevenção ao uso de drogas e assim foram direcionados a responder sobre as caraterísticas dos mesmos (Tabela 2). A maioria dos programas era aplicada sem regularidade e a oferta, quando ocorria, tinha duração média de um trimestre. Quase todos os programas foram oferecidos para os alunos e em menos da metade deles houve participação da família e da comunidade. Os dados mostram maior prevalência de oferta de programas de prevenção ao uso de drogas para alunos matriculados no ensino fundamental II ( $6^{\circ}$ ao $9^{\circ}$ ano). Observou-se que cerca de $40 \%$ implementaram programas desenvolvidos pela equipe da própria 
Tabela 1. Características dos respondentes e das escolas que participaram do estudo. Brasil, 2014 ( $\mathrm{n}=1.151)$.

\begin{tabular}{|c|c|c|c|}
\hline \multirow{3}{*}{ Variáveis } & \multicolumn{3}{|c|}{ Total } \\
\hline & \multicolumn{3}{|c|}{$\mathrm{n}=1.151$} \\
\hline & $\mathbf{n}$ & $\%$ & 95\%IC \\
\hline \multicolumn{4}{|l|}{ Características dos Respondentes } \\
\hline \multicolumn{4}{|l|}{ Sexo } \\
\hline Feminino & 855 & 74,4 & $71,8-76,9$ \\
\hline Masculino & 294 & 25,6 & $23,1-28,2$ \\
\hline \multicolumn{4}{|l|}{ Idade } \\
\hline $20-29$ anos & 55 & 4,8 & $3,6-6,2$ \\
\hline $30-39$ anos & 343 & 29,8 & $27,2-32,5$ \\
\hline $40-49$ anos & 471 & 40,9 & $38,1-43,8$ \\
\hline $50-59$ anos & 239 & 20,8 & $18,4-23,2$ \\
\hline $60-69$ anos & 43 & 3,7 & $2,7-5,0$ \\
\hline \multicolumn{4}{|l|}{ Escolaridade } \\
\hline Ensino Médio & 29 & 2,5 & $1,7-3,6$ \\
\hline Ensino Superior & 259 & 22,5 & $20,1-25,0$ \\
\hline Pós-graduação (Especialização) & 773 & 67,2 & $64,4-69,9$ \\
\hline Pós-graduação (Stricto Sensu) & 89 & 7,7 & $6,3-9,4$ \\
\hline \multicolumn{4}{|l|}{ Cargo } \\
\hline Diretor & 505 & 51,2 & $48,0-54,3$ \\
\hline Coordenador Pedagógico & 397 & 40,2 & $37,1-43,3$ \\
\hline Coordenador de Programa de Prevenção & 60 & 6,1 & $4,7-7,7$ \\
\hline Outros & 25 & 2,5 & $1,6-3,7$ \\
\hline Participou de curso sobre drogas & 739 & 65,1 & $62,2-67,8$ \\
\hline \multicolumn{4}{|l|}{ Características sociodemográficas das escolas } \\
\hline \multicolumn{4}{|l|}{ Rede } \\
\hline Pública & 893 & 77,6 & $75,1-80,0$ \\
\hline Privada & 258 & 22,4 & $20,0-24,9$ \\
\hline \multicolumn{4}{|l|}{ Região } \\
\hline Sudeste & 524 & 45,5 & $42,6-48,4$ \\
\hline Sul & 163 & 14,2 & $12,2-16,3$ \\
\hline Nordeste & 287 & 24,9 & $22,5-27,5$ \\
\hline Norte & 77 & 6,7 & $5,3-8,3$ \\
\hline Centro-oeste & 100 & 8,7 & $7,1-10,5$ \\
\hline \multicolumn{4}{|l|}{ Porte $^{1}$} \\
\hline Pequeno & 809 & 70,3 & $67,5-72,9$ \\
\hline Médio & 281 & 24,4 & $22,0-27,0$ \\
\hline Grande & 61 & 5,3 & $4,1-6,8$ \\
\hline \multicolumn{4}{|l|}{ Localização } \\
\hline Não Capital & 908 & 79,6 & $77,2-81,9$ \\
\hline Capital & 232 & 20,4 & $18,0-22,8$ \\
\hline
\end{tabular}

${ }^{1}$ Pequeno (até 800 alunos); Médio (801 até 1600 alunos); Grande (acima de 1600 alunos).

escola e cerca de $30 \%$ relataram que os programas foram oferecidos por instituições da área da saúde. A Polícia Militar se destaca como principal entidade promotora de programas nas escolas, estando presente em mais de 70\% das escolas que reportam possuir programas. Isso significa, ainda, que, na amostra completa, 35,7\% (411/1151) das escolas reportaram aplicar PROERD (Programa Educacional de Resistências às Drogas e Violência).

Ainda de acordo com a Tabela 2, pode-se observar que os programas de prevenção ao uso de drogas oferecidos nas escolas participantes do estudo combinavam mais de um modelo teórico. 
Tabela 2. Características dos programas de prevenção ao uso de drogas das escolas, Brasil, 2014 (n=580).

\begin{tabular}{|c|c|c|c|}
\hline \multirow{3}{*}{ Variáveis } & \multicolumn{3}{|c|}{ Total } \\
\hline & \multicolumn{3}{|c|}{$\mathrm{n}=\mathbf{5 8 0 ^ { 1 }}$} \\
\hline & $\mathbf{n}$ & $\%$ & 95\%IC \\
\hline \multicolumn{4}{|l|}{ Frequência das atividades } \\
\hline Esporádica (sem regularidade) & 340 & 58,6 & $54,5-62,7$ \\
\hline Sistemática (com regularidade) & 240 & 41,4 & $37,3-45,5$ \\
\hline \multicolumn{4}{|l|}{ Período de duração } \\
\hline Um ano & 65 & 11,6 & $9,1-14,5$ \\
\hline Um semestre & 116 & 20,7 & $17,4-24,3$ \\
\hline Um trimestre & 321 & 57,2 & $53,0-61,4$ \\
\hline Um mês & 26 & 4,6 & $3,0-6,7$ \\
\hline Menos que um mês & 33 & 5,9 & $4,1-8,2$ \\
\hline \multicolumn{4}{|l|}{ Público Alvo } \\
\hline Alunos & 555 & 97,4 & $95,7-98,5$ \\
\hline Professores & 267 & 46,8 & $42,7-51,0$ \\
\hline Pais & 230 & 40,4 & $36,3-44,5$ \\
\hline Comunidade & 180 & 31,6 & $27,8-35,6$ \\
\hline Funcionários em geral (não professores) & 184 & 32,3 & $28,5-36,3$ \\
\hline \multicolumn{4}{|l|}{ Nível de ensino oferecido } \\
\hline Ensino Fundamental I & 195 & 33,6 & $29,8-37,6$ \\
\hline Ensino Fundamental II & 380 & 65,5 & $61,5-69,4$ \\
\hline Ensino Médio & 253 & 43,6 & $39,5-47,8$ \\
\hline \multicolumn{4}{|l|}{ Entidades que promovem o programa } \\
\hline Polícia Militar & 411 & 73,4 & $69,5-77,0$ \\
\hline Equipe da própria escola & 238 & 42,5 & $38,4-46,7$ \\
\hline Instituição da área da saúde & 176 & 31,4 & $27,6-35,4$ \\
\hline Secretaria da educação & 101 & 18,0 & $14,9-21,5$ \\
\hline Grupos Religiosos & 55 & 9,8 & $7,5-12,6$ \\
\hline ONG'S não religiosas & 35 & 6,3 & $4,4-8,6$ \\
\hline \multicolumn{4}{|c|}{ Modelos em programas de prevenção ao uso de drogas } \\
\hline Educação para saúde & 515 & 90,5 & $87,8-92,8$ \\
\hline Educação afetiva & 501 & 88,5 & $85,6-91,0$ \\
\hline Conhecimento científico & 461 & 81,3 & $77,8-84,4$ \\
\hline Oferecimentos de alternativas & 389 & 69,1 & $65,1-72,9$ \\
\hline Habilidades pessoais e sociais & 386 & 68,1 & $64,1-71,9$ \\
\hline Treinamento para resistir & 383 & 67,1 & $63,0-70,9$ \\
\hline Amedrontamento & 253 & 45,1 & $40,9-49,3$ \\
\hline Pressão do grupo positiva & 159 & 28,4 & $24,7-32,4$ \\
\hline \multicolumn{4}{|c|}{ Atividades desenvolvidas nas escolas para prevenção ao uso de drogas } \\
\hline Palestras com convidados & 505 & 87,7 & $84,7-90,2$ \\
\hline Solicita aos alunos trabalhos escolares & 496 & 86,7 & $83,6-89,4$ \\
\hline Apresentação de filmes & 463 & 81,2 & $77,8-84,3$ \\
\hline Dinâmica de grupo & 340 & 70,0 & $65,7-74,0$ \\
\hline Fornece material pedagógico & 392 & 69,0 & $65,0-72,8$ \\
\hline Eventos especiais & 344 & 60,1 & $56,0-64,2$ \\
\hline Projetos Multidisciplinares & 339 & 59,3 & $55,1-63,3$ \\
\hline Teatro & 273 & 56,6 & $52,1-61,1$ \\
\hline Palestras com ex-usuários de drogas & 235 & 42,0 & $37,8-46,2$ \\
\hline Projeto ligado a uma única disciplina & 229 & 40,0 & $36,0-44,2$ \\
\hline Aulas curriculares & 196 & 34,3 & $30,4-38,3$ \\
\hline Questionários sobre o tema "drogas" & 169 & 35,4 & $31,1-39,9$ \\
\hline
\end{tabular}


Mais de $80 \%$ das escolas relataram aplicar programas que trabalhavam educação para a saúde, educação afetiva e conhecimento científico. O modelo de amedrontamento esteve presente em $45 \%$ das escolas.

Notou-se ainda que quase $90 \%$ das escolas realizavam, como atividade preventiva, palestras com convidados e cerca de $40 \%$ das escolas faziam palestras com ex-usuários. Projeção de filmes e solicitação de trabalhos sobre o tema drogas também eram atividades de alta prevalência nos programas (Tabela 2).

A Tabela 3 apresenta os princípios de boas práticas de prevenção das escolas que responderam possuir um programa de prevenção ao uso de drogas. Constatou-se que a maioria das escolas trabalhava fatores de risco e proteção, além de apresentar informações sobre as principais drogas conhecidas, de maneira ajustada à idade dos participantes.

Quando avaliado o número total de princípios de boas práticas de prevenção presentes em cada programa, os dirigentes reportaram em média 8,3 princípios, variando de 0 (mínimo) a 15 (máxima) e desvio padrão de 3,3 (dado não apresentado em tabela).

A Tabela 4 apresenta os fatores associados à maior quantidade de princípios de boas práticas de prevenção nos programas identificados no estudo. O modelo final demonstra que as escolas privadas possuíam $14 \%$ mais probabilidade de apresentar mais princípios de boas práticas em seus programas, quando comparadas às escolas públicas. Além disso, ser ofertado por uma equipe da própria escola ou por uma instituição da área de saúde ou pela secretaria de educação também aumentou a quantidade de princípios de boas práticas apresentados pelo programa.

\section{Discussão}

Os dados do presente estudo sugerem que a prevenção ao uso de drogas nas escolas brasileiras se operacionaliza sem regularidade e com duração média de um trimestre, dirigidas principalmente aos alunos do ensino fundamental II ( $6^{\circ}$ ao $9^{\circ}$ ano), com integração de diferentes modelos teóricos e promovidos principalmente pela Polícia Militar (PM). Os fatores associados à presença de maior quantidade de princípios de boas práticas de prevenção nos programas identificados foram: pertencer à rede privada de ensino e receber programas ofertados por equipe da própria escola; instituição da área de saúde ou secretaria de educação.

No presente estudo, observou-se que algumas características dos programas de prevenção ao

Tabela 3. Princípios de boas práticas de prevenção dos programas de prevenção ao uso de drogas desenvolvidos nas escolas, Brasil, $2014\left(\mathrm{n}=382^{1}\right)$.

\begin{tabular}{|c|c|c|c|}
\hline \multirow{3}{*}{ Princípios de boas práticas } & \multirow{2}{*}{\multicolumn{3}{|c|}{$\begin{array}{c}\text { Total } \\
\mathrm{n}=\mathbf{3 8 2}\end{array}$}} \\
\hline & & & \\
\hline & $\mathbf{N}$ & $\%$ & 95\%IC \\
\hline Fatores de riscos e proteção & 362 & 94,8 & $92,0-96,8$ \\
\hline Apresenta informações sobre as principais drogas conhecidas & 349 & 92,6 & $89,4-95,0$ \\
\hline Ajuste idade e características grupo & 284 & 76,3 & $71,7-80,6$ \\
\hline Ensino fundamental: treinamento de habilidades & 240 & 64,5 & $59,4-69,4$ \\
\hline Utiliza técnica interativas de atividades & 229 & 61,7 & $56,6-66,7$ \\
\hline Contínuo e de longo prazo & 221 & 59,4 & $54,2-64,4$ \\
\hline Participação da família & 192 & 51,3 & $46,1-56,5$ \\
\hline Combina mais de um modelo de prevenção (programa escolar, familiar e comunitário) & 184 & 50,8 & $45,5-56,1$ \\
\hline Ensino médio: habilidades para a resistência a oferta de drogas & 164 & 46,9 & $41,5-52,2$ \\
\hline Pré-escola (agressividade, habilidades sociais e dificuldades escolares) & 172 & 46,4 & $41,2-51,6$ \\
\hline Desenhado para os alunos da escola & 160 & 43,0 & $37,9-48,2$ \\
\hline Participação da comunidade & 144 & 38,7 & $33,7-43,9$ \\
\hline Baseado em outro programa cuja eficácia já foi comprovada cientificamente & 137 & 37,7 & $32,7-42,9$ \\
\hline Treinamento de professores & 119 & 32,2 & $27,4-37,2$ \\
\hline Reforço do programa em momentos de transição da vida escolar & 115 & 32,1 & $27,3-37,2$ \\
\hline
\end{tabular}

${ }^{1}$ Total de escolas que responderam ao módulo completo sobre princípios de boas práticas de prevenção nas escolas. Optou-se por não imputar as não-respostas. 
Tabela 4. Regressão de Poisson para contagem do número de princípios de boas práticas de prevenção nos programas identificados no estudo, de acordo com auto-relato dos entrevistados Brasil, 2014 ( $\mathrm{n}=318$ ).

\begin{tabular}{|c|c|c|c|c|c|c|}
\hline \multirow{2}{*}{ Variáveis } & \multicolumn{3}{|c|}{ Regressão univariada } & \multicolumn{3}{|c|}{ Regressão multivariada } \\
\hline & IRR & 95\%IC & p-valor & IRR & 95\%IC & p-valor \\
\hline \multicolumn{7}{|c|}{ Características demográficas das escolas } \\
\hline \multicolumn{7}{|l|}{ Rede } \\
\hline Pública & 1,00 & -- & -- & 1,00 & -- & -- \\
\hline Privada & 1,11 & $1,01-1,21$ & 0,030 & 1,14 & $1,03-1,25$ & 0,008 \\
\hline \multicolumn{7}{|l|}{ Região } \\
\hline Sudeste & 1,00 & -- & -- & -- & -- & -- \\
\hline Sul & 0,97 & $0,88-1,08$ & 0,645 & -- & -- & -- \\
\hline Nordeste & 1,01 & $0,90-1,12$ & 0,907 & -- & -- & -- \\
\hline Norte & 0,98 & $0,83-1,15$ & 0,793 & -- & -- & -- \\
\hline Centro-oeste & 1,04 & $0,90-1,19$ & 0,623 & -- & -- & -- \\
\hline \multicolumn{7}{|l|}{ Porte ${ }^{1}$} \\
\hline Pequeno & 1,00 & -- & -- & -- & -- & -- \\
\hline Médio & 1,10 & $1,01-1,20$ & 0,031 & -- & -- & -- \\
\hline Grande & 1,16 & $0,98-1,38$ & 0,074 & -- & -- & -- \\
\hline \multicolumn{7}{|l|}{ Localização } \\
\hline Capital & 1,00 & -- & -- & -- & -- & -- \\
\hline Não Capital & 1,04 & $0,94-1,14$ & 0,471 & -- & -- & -- \\
\hline \multicolumn{7}{|c|}{ Entidades que promovem o programa } \\
\hline Polícia Militar (Proerd) ${ }^{1}$ & 0,93 & $0,86-1,01$ & 0,092 & -- & -- & -- \\
\hline Equipe da própria escola ${ }^{1}$ & 1,22 & $1,13-1,32$ & $<0,001$ & 1,17 & $1,08-1,27$ & $<0,001$ \\
\hline Instituição da área da saúde ${ }^{1}$ & 1,17 & $1,08-1,27$ & $<0,001$ & 1,09 & $1,00-1,19$ & 0,048 \\
\hline Secretaria da educação ${ }^{1}$ & 1,21 & $1,10-1,32$ & $<0,001$ & 1,20 & $1,09-1,33$ & $<0,001$ \\
\hline $\mathrm{ONG}^{\prime} \mathrm{S}$ não religiosas ${ }^{1}$ & 1,12 & $0,98-1,28$ & 0,096 & -- & -- & -- \\
\hline Grupos Religiosos $^{1}$ & 1,09 & $0,96-1,24$ & 0,201 & -- & -- & -- \\
\hline
\end{tabular}

"Não" é a referência.

uso de drogas são semelhantes aos relatados por um estudo realizado em 79 escolas do município de São Paulo na década de $1980^{17}$. A maior parte dos dirigentes que participaram do estudo de Carlini-Cotrim e Rosemberg ${ }^{17}$ relatou que os programas ocorriam com regularidade esporádica, sendo promovidos por entidades não educacionais e oferecidos principalmente aos alunos. Esse resultado sugere que as escolas brasileiras fizeram poucos progressos na direção da implementação de currículos de prevenção do uso de drogas baseados evidências nas últimas três décadas.

Estudos epidemiológicos realizados nos Estados Unidos (EUA) sobre a prevalência de implantação de programas de prevenção ao uso de drogas nas escolas mostraram que o progresso para implementação de currículos com evidências científicas comprovadas foi gradativo e lento ${ }^{5,18-21}$. Esse progresso ocorreu com investimentos, medidas de políticas públicas consistentes e através de registro nacional de programas e práticas baseadas em evidência em prevenção ao uso de dro- gas $^{19,20}$. No Brasil, a prevenção ao uso de drogas faz parte de um dos componentes de ação do Programa Saúde na Escola (PSE), uma parceria dos Ministérios da Saúde e da Educação, implantado nas escolas públicas, por meio de suporte do SUS, via Atenção Básica ${ }^{22}$, além de fazer parte do capítulo 4 da Política Nacional sobre Drogas ${ }^{23}$.

Constatou-se neste estudo baixa inclusão dos familiares como público dos programas, sendo os alunos $(94,0 \%)$ o principal público-alvo deles. Tal achado sugere que a maioria dos programas não contempla currículos integrados com a comunidade e família, prática de intervenção que seria necessária em programas eficazes de prevenção ao uso de drogas por adolescentes ${ }^{24-27}$.

Um trimestre foi o período médio de implantação dos programas. Esse período pode ser considerado adequado dependendo do conteúdo trabalhado ${ }^{9}$. A literatura científica recomenda que o período das intervenções do programa ocorra em 10 a 15 seções estruturadas para atingir resultados positivos na prevenção ao uso de 
$\operatorname{drogas}^{12}$. Dessa forma, considerando-se encontros semanais, espera-se um programa que ocorra no período de 3 a 4 meses.

Foi observado neste estudo que a PM é a principal entidade promotora de programas nas escolas brasileiras. O programa oferecido pela PM é o PROERD que tinha por base, até 2014, ano em que os dados do presente estudo foram coletados, o Projeto DARE (Drug Abuse Resistance Education), desenvolvido pelo Departamento de Polícia dos EUA ${ }^{28}$. A avaliação do programa DARE nos EUA evidenciou que o programa não foi eficaz na prevenção ao uso de drogas por adolescentes ${ }^{29-32}$.

Observou-se ainda que programas de prevenção ao uso de drogas oferecidos nas escolas brasileiras combinavam mais de um modelo teórico. Estudos sugerem que os programas que combinam vários modelos de prevenção foram significativamente mais eficazes do que aqueles baseados somente em um modelo ${ }^{23,33}$.

Apesar de a maioria das escolas incluir modelos indicados pela literatura científica em seus programas, $45 \%$ das escolas ainda se utilizam do modelo de amedrontamento, que têm sido apontado como ineficaz na prevenção ao uso de drogas em adolescentes ${ }^{10}$. A presença de ex-usuários de drogas nas atividades preventivas das escolas sugere a ausência de embasamento científico na prática preventiva de $40 \%$ das escolas, de que essa técnica preventiva está associada à ausência de efeito ou mesmo a resultados negativos ${ }^{12,27}$.

Observou-se que a maioria das escolas realizava palestras com convidados, solicitava trabalhos, apresentava filmes sobre drogas, fornecia material, tratava do tema drogas em eventos especiais e fazia dinâmicas de grupo com os alunos. Entretanto, não ficou claro nos resultados do presente estudo, se essas atividades são ações isoladas ou ações integradas. Contudo, programas baseados em boas práticas em prevenção na escola proporcionam experiências práticas para os participantes, ao contrário de programas que oferecem apenas informações e discussão, permitindo que os alunos desenvolvam e pratiquem novas habilidades através de atividades interativas ${ }^{8}$.

Em relação às boas práticas de prevenção, os dirigentes relataram em média 8,3 dos 15 sugeridos pelo NIDA $^{11}$ presentes nos programas de prevenção ao uso de drogas das escolas participantes do estudo. As escolas trabalhavam em seus programas os fatores de risco e proteção, apresentavam informações sobre as principais drogas, ofereciam treinamentos de habilidades no ensino fundamental e utilizavam técnicas in- terativas. Porém, observou-se que mais de $60 \%$ dos programas não foram baseados em outros programas cuja eficácia foi comprovada, não envolveram treinamento de professores e não foi oferecido reforço do programa em momentos de transição da vida escolar, sugerindo falha de aspectos importantes de boas práticas em prevenção ${ }^{12}$.

A maior quantidade de princípios de boas práticas de prevenção foi identificada em escolas privadas, o que pode ser consequência da disponibilidade de mais recursos financeiros para investimento em formação dos profissionais e compra de material adequado. O estudo realizado com 263 dirigentes de escolas do município de São Paulo sobre fatores associados à implementação de programas de prevenção ao uso de drogas, mostrou que as restrições econômicas são um grande desafio no processo de implantação na rede pública de ensino ${ }^{16}$.

Por fim, foi observado que programas ofertados por uma equipe da própria escola ou por uma instituição da área de saúde ou pela secretaria de educação aumentou a chance de apresentarem mais princípios de boas práticas. Esse resultado evidencia a importância do envolvimento da equipe da escola e do apoio da secretaria da educação na prática de prevenção ao uso de drogas para o desenvolvimento de atividades baseadas nos princípios de boas práticas em prevenção. Assim, a expansão das ações do $\operatorname{PSE}^{34}$ pode contribuir para o avanço na prática de prevenção baseada em evidencia nas escolas brasileiras.

Como limitações do estudo, destaca-se a dificuldade em se conseguir contato com $25 \%$ das escolas sorteadas, o que acabou reduzindo a possibilidade de generalização dos achados. Outra limitação diz respeito ao instrumento fechado e de autopreenchimento, que não permitiu investigarmos com mais detalhes os programas que estão sendo implementados nas escolas e a compreensão exata das perguntas pelos respondentes. Idealmente, teríamos feito visitas para observar os programas de prevenção em todas as escolas, mas tais métodos estão além do escopo deste estudo. Apesar das limitações, esta é a primeira tentativa de avaliar as características dos programas em amostra probabilística de escolas brasileiras.

\section{Conclusão}

A prática de prevenção ao uso drogas nas escolas brasileiras necessita de aperfeiçoamento de ações. Os programas são realizados através de 
atividades que não se baseiam necessariamente nos princípios de boas práticas de prevenção. É de suma importância que os responsáveis pela adoção de um programa de prevenção escolar se orientem pelas recomendações de boas práticas, a fim de que as ações desenvolvidas sigam evidências científicas de sucesso. A oferta de cursos de qualidade que instrumentalizem os profissionais do campo da prevenção, a elaboração de diretrizes nacionais de boas práticas em prevenção ao uso de drogas e a implantação de políticas públicas baseadas em evidências podem contribuir para garantir que os dirigentes das escolas brasileiras possam implantar um programa eficaz na prevenção ao uso de drogas e que se enquadre na proposta pedagógica de cada escola. Por fim, futuros estudos devem ser planejados para avaliar a eficácia e a efetividade desses programas, contribuindo na decisão dos dirigentes para a adoção de programas de melhor custo-efetividade.

\section{Colaboradores}

APD Pereira colaborou na concepção do projeto, coleta e análise dos dados e redação do artigo. ZM Sanchez foi responsável pelo desenho do estudo, supervisão da análise dos dados e revisão do artigo. 


\section{Referências}

1. Zavela KJ. Developing effective school-based drug abuse prevention programs. Am J Health Behav 2002; 26(4):252-265.

2. Faggiano F, Vigna-Taglianti FD, Versino E, Zambon A, Borraccino A, Lemma P. School-based prevention for illicit drugs use: A systematic review. Prev Med 2008; 46(5):385-396.

3. Hale DR, Fitzgerald-Yau N, Viner RM. A systematic review of effective interventions for reducing multiple health risk behaviors in adolescence. Am J Public Health 2014; 104(5):e19-41.

4. Foxcroft DR, Tsertsvadze A. Universal school-based prevention programs for alcohol misuse in young people. Cochrane Database Syst Rev 2011; 5:CD009113.

5. LeNoue SR, Riggs PD. Substance Abuse Prevention. Child Adolesc Psychiatr Clin N Am 2016; 25(2):297305.

6. Werch CE, Owen DM. Iatrogenic effects of alcohol and drug prevention programs. J Stud Alcohol 2002; 63(5):581-590.

7. Sloboda Z, Pyakuryal A, Stephens PC, Teasdale B, Forrest D, Stephens RC, Grey SF. Reports of substance abuse prevention programming available in schools. Prev Sci 2008; 9(4):276-287.

8. Thom B. Good practice in school based alcohol education programmes. Patient Educ Couns 2017; 100 (Supl. 1):S17-S23.

9. Nation M, Crusto C, Wandersman A, Kumpfer KL, Seybolt D, Morrissey-Kane E, Davino K. What works in prevention: Principles of effective prevention programs. Am Psychol 2003; 58(6-7):449.

10. Peters LW, Kok G, Ten Dam GT, Buijs GJ, Paulussen TG. Effective elements of school health promotion across behavioral domains: a systematic review of reviews. BMC Public Health 2009; 9:182.

11. National Institute on Drug Abuse (NIDA). Preventing drug use among children and adolescents: A research-based guide for parents, educators, and community leaders. North Bethesda: Diane Publishing; 2003.

12. United Nations Office on Drugs and Crime (UNODC). International Standards on drug Use Prevention. Viena: UNODC; 2013.

13. Granello DH, Wheaton JE. Online data collection: Strategies for research. J Counseling Development 2004; 82(4):387-393.

14. Andrews D, Nonnecke B, Preece J. Electronic survey methodology: A case study in reaching hard-to-involve Internet users. Int J Human-Computer Interaction 2003; 16(2):185-210.

15. Ringwalt CL, Ennett S, Vincus A, Thorne J, Rohrbach LA, Simons-Rudolph A. The prevalence of effective substance use prevention curricula in U.S. middle schools. Prev Sci 2002; 3(4):257-265.

16. Pereira AP, Paes Â, Sanchez ZM. Factors associated with the implementation of programs for drug abuse prevention in schools. Rev Saude Publica 2016; 50:44.

17. Carlini-Cotrim B, Rosemberg F. Drogas: prevenção no cotidiano escolar. Cad Pesquisa 1990; (74):40-46.
18. Hallfors D, Godette D. Will the Principles of Effectiveness' improve prevention practice? Early findings from a diffusion study. Health Educ Res 2002; 17(4):461-470.

19. Ringwalt C, Hanley S, Vincus AA, Ennett ST, Rohrbach LA, Bowling JM. The prevalence of effective substance use prevention curricula in the nation's high schools. J Prim Prev 2008; 29(6):479-488.

20. Ringwalt C, Vincus AA, Hanley S, Ennett ST, Bowling JM, Haws S. The prevalence of evidence-based drug use prevention curricula in U.S. middle schools in 2008. Prev Sci 2011; 12(1):63-69.

21. Hanley SM, Ringwalt C, Ennett ST, Vincus AA, Bowling JM, Haws SW, Rohrbach LA. The prevalence of evidence-based substance use prevention curricula in the nation's elementary schools. J Drug Educ 2010; 40(1):51-60.

22. Brasil. Decreto $n^{\circ}$ 6.286, de 5 de dezembro de 2007. Institui o Programa Saúde na Escola-PSE, e dá outras providências. Diário Oficial da União; 2007.

23. Brasil. Resolução no 03/GSIPR/CH/CONAD, de 27 de outubro de 2005. Aprova a Política Nacional sobre Drogas. Diário Oficial da União; 2005.

24. Das JK, Salam RA, Arshad A, Finkelstein Y, Bhutta ZA. Interventions for Adolescent Substance Abuse: An Overview of Systematic Reviews. J Adolesc Health 2016; 59(4S):S61-S75.

25. Kuntsche S, Kuntsche E. Parent-based interventions for preventing or reducing adolescent substance use - A systematic literature review. Clin Psychol Rev 2016; 45:89-101.

26. Foxcroft DR, Tsertsvadze A. Universal family-based prevention programs for alcohol misuse in young people. Cochrane Database Syst Rev 2011; 9.

27. Griffin KW, Botvin GJ. Evidence-based interventions for preventing substance use disorders in adolescents. Child Adolesc Psychiatr Clin N Am 2010; 19(3):505526.

28. Shamblen SR, Courser MW, Abadi MH, Johnson KW, Young L, Browne TJ. An international evaluation of DARE in São Paulo, Brazil. Drugs Educ Prev Policy 2014; 21(2):110-119.

29. Pan W, Bai H. A multivariate approach to a meta-analytic review of the effectiveness of the DARE program. Int J Environ Res Public Health 2009; 6(1):267-277.

30. Vincus AA, Ringwalt C, Harris MS, Shamblen SR. A short-term, quasi-experimental evaluation of DARE's revised elementary school curriculum. J Drug Educ 2010; 40(1):37-49.

31. West SL, O’Neal KK. Project DARE outcome effectiveness revisited. Am J Public Health 2004; 94(6):10271029.

32. Perry CL, Komro KA, Veblen-Mortenson S, Bosma LM, Farbakhsh K, Munson KA, Stigler MH, Lytle LA. A randomized controlled trial of the middle and junior high school DARE and DARE Plus programs. Arch Pediatr Adolesc Med 2003; 157(2):178-184. 
33. Foxcroft DR, Tsertsvadze A. Universal multi-component prevention programs for alcohol misuse in young people. Cochrane Database Syst Rev 2011; (9):CD009307.

34. Sousa MC, Esperidião MA, Medina MG. Intersectorality in the 'Health in Schools' Program: an evaluation of the political-management process and working practices. Cien Saude Colet 2017; 22(6):1781-1790.

Artigo apresentado em 22/06/2018

Aprovado em 05/11/2018

Versão final apresentada em 07/11/2018 